\title{
Analysis of Microbial Biochemical Inducting Nutritional Contents in Barley
} Anam Yousaf', Yaseen Ashraf ${ }^{2 *}$, Nasim Ahmad Yasin², Asma Ibrahim², Aqeel Ahmad², Waheed Ullah Khan', Zoobia Bashir ${ }^{4}$, Waheed Akram²,3 and Zarish Noreen ${ }^{5}$

${ }^{1}$ College of Earth and Environmental Sciences, University of the Punjab, Lahore, Pakistan

${ }^{2}$ Institute of Agricultural Sciences, University of the Punjab, Lahore, Pakistan

${ }^{3}$ Institute for Medicinal Plants, College of Plant Science and Technology, Huazhong Agricultural University, Wuhan, China

${ }^{4}$ Department of Physics, University of the Punjab, Lahore, Pakistan

${ }^{5}$ Healthcare Biotechnology Atta ur Rahman School of Applied Sciences, National University of Science and Technology, H-12 Campus, Islamabad, Pakistan

\begin{abstract}
Current study focuses the biochemical analysis of the metabolites of Acetobacter aceti to explore bioactive compounds inducing nutritional elements (including vitamins) in barley seeds. Thirteen chemical compounds were isolated using bioactivity guided assay and chromatographic analyses of bacterial extracts. The, principal component analysis (PCA) indicated the most active biochemicals among them. It was resulted into five chemical compounds (i.e. Mevalonic Acid, Quinolinic Acid, Pyridoxic Acid, p.Aminobenzoate and and a-Oxobutanoic acid), which were highly associated with increased quantities of phytosterols, phenols and tested vitamins. A solvent system consisting upon chloroform:ethanol (4:1) was recommended as the best solvent system for extraction of active compounds from bacterial metabolites. Moreover, the mechanisms have been proposed according to which plant pathways were directed towards elevated biosynthesis of nutritional elements. Significant quantities of Mevalonic Acid were concluded into induction of isoprenoid pathway. Current investigation provides is a step towards biochemical interactions inside plant cell.
\end{abstract}

Keywords: Bioactivity guided assay; Chromatography; Fractionation; Mass spectrometery; Precursor

\section{Introduction}

Alteration in physiological pathways and processes of plants under the influence of microbial treatments is a well-known phenomenon in plant sciences. Plant scientists have successfully utilized this phenomenon to achieve numerous goals related to plant protection $[1,2]$. It has been concluded that biological treatments bring large scale changes in plant metabolism. They alter the rate of plant metabolic pathways, which result into alteration in biochemical contents of plant tissues [3-5]. Thus, a number of studies have reported changes in quantities of biochemicals in plants which are intrinsically very diverse from one another [6,7]. The potential of microbes (increase the nutritional contents of barley seeds) has been studied in Fungal Biotechnology Lab, Institute of Agricultural Sciences, University of the Punjab, Lahore. Nutritional contents were primarily focused in current investigation, which exhibit prime importance in defining food quality. The advancements in the field of elevation of water soluble vitamin contents and the identification of biochemical inducers responsible for that elevation [8]. Current study evaluated a bacterial species (Acetobacter aceti), which altered metabolic processes of plant in such way that nutritional elements of barley seeds were elevated. However, the bacterial species has been rarely reported as a potential pathogen of pink disease of pineapple [9]. Thus, cannot be applied carelessly in agriculture fields. Therefore, it is imperative to determine its bioactive compounds, which could be applied to get improved quality barley produce. Thus, current investigation mainly focuses upon screening of bacterial metabolites to identify the specific biochemical, which would help to improve the quality of agricultural produce.

\section{Materials and Methods}

\section{Procurement of bacterial species and barley strains}

Bacterial species, i.e., A. aceti (FCBP-537) was procured from Fungal Culture Bank of Pakistan, University of the Punjab, Lahore, Pakistan. Its cultures were maintained on Petriplates containing LB agar cultures medium. Barley seeds were procured from Fungal Biotechnology Lab and grown in plastic pots under greenhouse conditions $\left(22 \pm 2^{\circ} \mathrm{C}\right)$ These plants were used to perform biological assay at the age of two weeks. Bacterial species was grown in $4 \mathrm{~L}$ of Czapek Dox broth medium based upon sucrose (3\%). Bacterial cells were removed by centrifugation leaving behind the cell free culture filtrate (CFCF), which was subjected to organic solvent extraction.

\section{Organic solvent extraction}

CFCF was evaporated using rotary evaporator at temperature $40^{\circ} \mathrm{C}$ up to $1 \mathrm{~L}$ of remaining volume. Then, it was successively extracted with n- hexane, chloroform, ethyl acetate and n- butanol. Former fraction of each organic solvent was separated before starting extraction process with next solvent. Thus, organic fractions of all solvents were prepared and evaporated yielding dry extracts. These dried fractions were checked for their ability to induce vitamin quantities in barley, and the best performing fraction was screened out for further downstream purification steps. This purification was carried out through chromatographic techniques, while checking the bioactivity of all sub-fractions at every downstream level. Hence, the process termed as 'bioactivity guided isolation of active compounds.'

\section{Bioactivity guided isolation of active compounds}

Crude fraction exhibiting the maximum bioactivity was processed through column chromatography generating five sub-fractions, i.e., SF01, SF02, SF03..., SF05. The biochemical profile of each sub-fraction

${ }^{*}$ Corresponding author: Yaseen Ashraf, Institute of Agricultural Sciences, University of the Punjab, Lahore, Pakistan, Tel: +92 3074826093; E-mail: yaseenashraf00@gmail.com

Received July 25, 2016; Accepted August 21, 2016; Published August 28, 2016

Citation: Yousaf A, Ashraf Y, Yasin NA, Ibrahim A, Ahmad A, et al. (2016) Analysis of Microbial Biochemical Inducting Nutritional Contents in Barley. J Microb Biochem Technol 8: 395-403. doi: 10.4172/1948-5948.1000315

Copyright: $\odot 2016$ Yousaf $A$, et al. This is an open-access article distributed under the terms of the Creative Commons Attribution License, which permits unrestricted use, distribution, and reproduction in any medium, provided the original author and source are credited. 
was estimated through thin layer chromatography, while bioactivity was studied by applying sub-fractions on barley plants.

Organic sub-fraction of TLC, induced maximum quantities in barley was analyzed through chromatographic techniques to identify specific compound/s responsible for vitamin induction under greenhouse assay. Organic fraction was further fractionated into 5 sub-fractions (i.e., FR01 20\%, FR02 40\%, FR03 60\%, FR04 80\% and FR05 100\% through Column Chromatography (CC) and all sub-fractions were studied for vitamin elevation potential, through bioactivity guided assay. The solvent used in the preparation of those sub-fractions was ethanol: chloroform, with constant increment of $20 \%$ in concentration of chloroform. Moreover, Preparative Thin Layer Chromatography (PTLC) was performed to remove maximum possible contaminants from the fractions. PTLC was performed through glass plates $(20 \times 20 \mathrm{~cm})$ coated with silica. Bioactive compounds were tried to be isolated with minimum possible number of contaminants before performing Gas Chromatography Mass Spectrometery (GCMS).

\section{Gas chromatography mass spectrometery}

GCMS was performed on gas chromatography unit of Agilent Technologies (7890A), accompanied with mass spectrometer unit of $(5975 \mathrm{C})$ and column HP-5MS. Operation was started from $70^{\circ} \mathrm{C}$ temperature with continuous increment of $5^{\circ} \mathrm{C} / \mathrm{min}$ up to $200^{\circ} \mathrm{C}$ temperature. Operation was further continued up to $240^{\circ} \mathrm{C}$ at a continuous increment of $10^{\circ} \mathrm{C} / \mathrm{min}$. The flow of rate of carrier gas (Helium) was maintained $1 \mathrm{~mL} / \mathrm{min}$ during the entire operation. Mass spectra of compounds were identified using TURBOMASS (Perkinelmer, Shelton, USA) equipped with mass spectral NIST library.

\section{Activity verification of identified compound}

Thus, purified chemicals were purchased from Sigma Aldrich and applied separately to sets of barley plants of two weeks of age, treating the control with distilled sterilized water. Seeds of the plants were tested for vitamin contents and data were compared test the efficacy of treatment. The whole experiment was independently repeated thrice to ensure the authenticity of the results.

\section{Determination of mechanism}

Plants treated with bioactive compounds were subjected to total metabolomics analysis through GCMS including a negative control treatment (with no chemical appication). For this purpose total metabolites extracts of the plant were derivative thought Methoxyamine hydrochloride (MOX) reagents and N-Trimethysilyl trifluoroacetamide (MSTFA) reagents, providing overnight and 30 min incubation at room temperature, respectively. Other GCMS conditions were same as described earlier. However, this time data were recorded regarding the change in metabolites of plants due to application of bioactive compounds of $A$. aceti.

\section{Data analysis}

Statistical analysis of data was carried out through Analysis of Variance (ANOVA) and Duncan's Multiple Range Test (DMRT) at $p$ $\leq 0.05$. Statistical operations were performed using statistical package of MS-Excel based add-in DSAASTAT (Onofri, Italy). Principle component analysis (PCA) was performed to determine the most active bacterial metabolite, and screened chemical was applied to barley plants to verify the results of the study.

\section{Results}

Chloroform fraction provided the maximum levels of most of the tested nutritional elements. Significantly higher quantities of Ascorbic acid, Pyridoxine, Folic acid and Riboflavin were recorded in barley; however, Niacin was recorded in equal quantities in both treatments of chloroform and ethyl acetate. Phytosterols, Falvonoids, Saponins and Triterpenoids were also recorded maximum under the activity of chloroform fraction. Ethyl acetate had non-significantly higher contents of Panthothenic acid than chloroform treatment. Whereas, n-Butanol exhibited non-significantly more amounts of Thiamine contents than chloroform treatment. n-Hexane was recorded with non-significantly lesser quantities of Panthothenic acid and Thiamine contents than respective values of control treatment (Table 1).

Maximum quantities of nutritional contents were found in $80 \%$ chloroform treatment. It induced Ascorbic Acid most efficiently in barley. Moreover, it also significantly induced the highest contents of

\begin{tabular}{|c|c|c|c|c|c|c|}
\hline Nutritional Element & Chloroform & n-butanol & Ethyl Acetate & n-hexane & n-heptane & Control \\
\hline ascorbic acid & $0.074 \mathrm{a}$ & $0.042 d$ & $0.037 \mathrm{de}$ & $0.049 \mathrm{c}$ & $0.056 \mathrm{~b}$ & $0.028 \mathrm{f}$ \\
\hline niacin & $0.051 \mathrm{a}$ & $0.044 a b$ & $0.051 \mathrm{a}$ & $0.038 b$ & $0.031 \mathrm{bc}$ & $0.023 \mathrm{c}$ \\
\hline panthothenic acid & $0.037 \mathrm{ab}$ & $0.029 \mathrm{bc}$ & $0.039 a$ & $0.016 \mathrm{~d}$ & $0.034 \mathrm{~b}$ & $0.017 \mathrm{~d}$ \\
\hline pyrodoxine & $0.049 a$ & $0.017 \mathrm{~d}$ & $0.016 \mathrm{~d}$ & $0.028 \mathrm{c}$ & $0.037 \mathrm{~b}$ & $0.018 \mathrm{e}$ \\
\hline thiamine & $0.016 a$ & $0.017 \mathrm{a}$ & $0.008 \mathrm{~b}$ & $0.006 \mathrm{c}$ & $0.009 \mathrm{~b}$ & $0.007 \mathrm{bc}$ \\
\hline folic acid & $0.014 \mathrm{a}$ & $0.006 \mathrm{c}$ & $0.007 \mathrm{bc}$ & $0.008 \mathrm{bc}$ & $0.009 \mathrm{~b}$ & $0.005 \mathrm{~cd}$ \\
\hline riboflavin & $0.065 a$ & $0.036 \mathrm{bc}$ & $0.041 \mathrm{~b}$ & $0.027 \mathrm{~cd}$ & $0.031 \mathrm{c}$ & $0.021 \mathrm{~d}$ \\
\hline pyhtosterol & $0.009 a$ & $0.008 \mathrm{~b}$ & $0.008 \mathrm{~b}$ & $0.005 \mathrm{~cd}$ & $0.006 \mathrm{c}$ & $0.004 \mathrm{~d}$ \\
\hline tannins & $0.026 \mathrm{c}$ & $0.029 \mathrm{~b}$ & $0.034 \mathrm{a}$ & $0.017 \mathrm{~d}$ & $0.013 \mathrm{e}$ & $0.016 \mathrm{~d}$ \\
\hline coumarins & $0.042 \mathrm{c}$ & $0.051 \mathrm{a}$ & $0.049 \mathrm{~b}$ & $0.031 \mathrm{~d}$ & $0.032 \mathrm{~d}$ & $0.030 \mathrm{~d}$ \\
\hline flavonoids & $0.009 a$ & $0.003 \mathrm{c}$ & $0.004 \mathrm{c}$ & $0.006 \mathrm{~b}$ & $0.002 \mathrm{~cd}$ & $0.007 b$ \\
\hline alkaloids & $0.003 d$ & $0.005 \mathrm{c}$ & $0.006 \mathrm{~b}$ & $0.007 \mathrm{ab}$ & $0.008 a$ & $0.005 \mathrm{c}$ \\
\hline phenols & $0.004 \mathrm{c}$ & $0.002 \mathrm{~d}$ & $0.005 b$ & $0.008 \mathrm{a}$ & $0.004 \mathrm{c}$ & $0.006 \mathrm{~b}$ \\
\hline saponins & $0.006 \mathrm{a}$ & $0.003 \mathrm{c}$ & $0.004 \mathrm{~b}$ & $0.003 \mathrm{c}$ & $0.002 \mathrm{c}$ & $0.004 \mathrm{~b}$ \\
\hline pectins & $0.002 \mathrm{~d}$ & $0.005 a b$ & $0.004 \mathrm{c}$ & $0.006 \mathrm{a}$ & $0.005 a b$ & $0.007 \mathrm{a}$ \\
\hline triterpenoids & $0.054 \mathrm{a}$ & $0.027 \mathrm{c}$ & $0.037 \mathrm{~b}$ & $0.030 \mathrm{c}$ & $0.018 \mathrm{~d}$ & $0.016 \mathrm{~d}$ \\
\hline
\end{tabular}

Data were statistically analyzed through Analysis of Variance and Duncan's Multiple Range Test using DSAASTAT (Onofri, Italy). Each nutritional element was compared along its own quantities extracted with different organic solvents. Each horizontal change in alphabet represents significant difference calculated at $p \geq 0.05$.

Table 1: Quantification of nutritional contents $(\mathrm{g} / \mathrm{Kg})$ of barley seeds, after treating barley plants with different organic solvent fractions of $A c e t o b a c t e r$ aceti extracts. 
Phytosterols, Phenols, Falvonoids, Saponins and Pectins. The quantity of Riboflavin was also elevated by $80 \%$ chloroform in the most efficient way. Pyrodixine was recorded to be significantly elevated after getting $80 \%$ chloroform treatment. Similarly, quantity of Niacin was found to be significantly higher in $80 \%$ chloroform than all other treatments. The second most efficient treatment in terms of nutritional elements induction was $100 \%$ chloroform. It induced Ascorbic acid significantly higher concentrations than 20, 40 and $60 \%$ chloroform treatments. Induction in the quantity of Pyrodoxine was also significantly higher in $100 \%$ chloroform treatment than 20,40 and $60 \%$. Similar trend was found in case of Riboflavin which was detected in significantly higher amounts in $100 \%$ chloroform treatments than 20, 40 and $60 \%$ treatments. Furthermore, second highest quantities of Flavonoids, Alkaloids, Phenols, Saponins and Pectins were also recorded under the influence of 100 chloroform application. Thiamine was the only vitamin, which did not show any significant variation in its quantities among all treatments; however, quantity of Folic acid was recorded to be significantly greater in $80 \%$ treatment with comparison to respective quantities in 20,40 and $60 \%$ treatment (Table 2). Two fractions $80 \%$ and $100 \%$ showed the overall efficient results, thus those two fractions were analyzed by GCMS to figure out active compound/s.

Thirteen chemical compounds were detected in the most active $(80 \%)$ chloroform fraction. These chemicals were identified as Thymol, Eugenol, Globulol, Mevalonic Acid, Quinolinic Acid, E-8Octadecacen-1-ol Acetate (ODCA), Pentadenoic Acid, Phthalic Ester, Octadecatrienoic Acid (ODTA), Pyridoxic Acid, $p$-Aminobenzoate, a-Oxobutanoic acid and Glycine. The second most active (100\%) chloroform fraction was missing in Quinolinic Acid and ODCA. However, the concentration of most of the compounds was retarded $100 \%$ chloroform fraction than $80 \%$ chloroform fraction, i.e., Quinolinic Acid, ODCA, Pentadenoic Acid, Phthalic Ester, ODTA, $p$-Aminobenzoate and $\alpha$-Oxobutanoic acid (Figure 1).

Chemical characteristics of all detected compounds were recorded in order to perform principle component analysis (PCA). All thirteen compounds had sufficiently apart peaks with good resolution. Thymol was the first compound detected at $8.03 \mathrm{~min}$ of chromatographic run with a peak area of $4.7 \mathrm{E}$. Eugenol was eluted at $8.88 \mathrm{~min}$ and recorded a peak area of 4.9E. Mass spectra of Globulol (peak area: 5.1E) and Mevalonic Acid (peak area: 4.3E) were recorded after chromatographic run of 9.17 and $9.29 \mathrm{~min}$, respectively. Quinolinic Acid with a peak area of $4.7 \mathrm{E}$ was detected at $12.33 \mathrm{~min}$, whereas, ODCA with a peak area of 5.6E was eluted at $13.68 \mathrm{~min}$. Pentadenoic Acid (5.8E) was eluted at $13.85 \mathrm{~min}$, whereas Phthalic Ester (5.9E) eluted at $14.08 \mathrm{~min}$. ODTA eluted at $15.75 \mathrm{~min}$ had the third largest peak area of $6.1 \mathrm{E}$, however Pyridoxic Acid (19.67 min) exhibited the highest peak area 7.4E. The second highest peak area (6.5E) was exhibited by $p$-Aminobenzoate, which had the retention time of $20.41 \mathrm{~min}$. $\alpha$-Oxobutanoic acid and Glycine (peak area: $5.3 \mathrm{E}$ and $4.2 \mathrm{E}$, respectively) were the last eluting compounds with the retention time of 21.18 and $21.28 \mathrm{~min}$, respectively (Tables 3 and 4).

p-Aminobenzoate and $\alpha$-Oxobutanoic acid were the compounds having maximum affinity with induction of nutrition contents. Moreover, Pyridoxic Acid had a third strongest association with induction of nutritional elements; and Quinolinic Acid recorded the fouth strongest relation in this hierarchy, just stronger than Mevalonic Acid. All these five compounds (i.e., Quinolinic Acid, $p$-Aminobenzoate, Pyridoxic Acid and $\alpha$-Oxobutanoic acid) collectively construct a group ( $\beta$ ), and are closely placed over a PCA plot. Similarly, Thymol, Globulol and ODCA recorded more variable association with chloroform concentration than with vitamin induction. Phthalic Ester was the least associated compound amond all thirteen biochemicals. Eugenol, Pentadenoic Acid, Thymol, ODTA and Glycine also exhibited weak associations with induction of nutritional contents (Figure 2).

Mass Spectra of bioactive compounds were recorded through TURBOMASS (Perkinelmer, Shelton, USA) and compared their respective compounds in NIST Library. More than 800 similarity scores were calculated in case of all identified compounds (Figures 3 and 4).

\section{Activity Verification of Identified Compounds}

$p$-Aminobenzoate induced maximum quantities of the nutritional contents. It recorded significantly higher quantities of Tannins, Flavonoids, Alkaloids, Saponins and six vitamins, i.e., Ascorbic acid, Niacin, Pathothenic acid, Pyridoxine, Thiamine and Folic acid However, The highest Quantities of Riboflavin were recorded under the

\begin{tabular}{|c|c|c|c|c|c|}
\hline & $20 \%$ & $40 \%$ & $60 \%$ & $80 \%$ & $100 \%$ \\
\hline ascorbic acid & 0.036 ef & $0.039 \mathrm{e}$ & $0.045 d$ & $0.074 \mathrm{a}$ & $0.057 b$ \\
\hline niacin & $0.025 \mathrm{gh}$ & $0.027 \mathrm{~g}$ & $0.031 \mathrm{fg}$ & $0.057 \mathrm{bc}$ & $0.044 \mathrm{de}$ \\
\hline panthothenic acid & $0.028 \mathrm{~g}$ & $0.029 \mathrm{~g}$ & $0.026 \mathrm{fg}$ & $0.031 \mathrm{fg}$ & $0.03 \mathrm{~g}$ \\
\hline pyrodoxine & $0.037 \mathrm{e}$ & 0.043 de & $0.046 \mathrm{~d}$ & $0.059 \mathrm{~b}$ & $0.051 \mathrm{c}$ \\
\hline thiamine & $0.013 \mathrm{ij}$ & $0.012 \mathrm{ij}$ & $0.016 \mathrm{ij}$ & $0.018 \mathrm{hi}$ & $0.015 \mathrm{i}$ \\
\hline folic acid & $0.007 \mathrm{j}$ & $0.009 \mathrm{j}$ & $0.013 \mathrm{j}$ & $0.015 \mathrm{i}$ & $0.011 \mathrm{ij}$ \\
\hline riboflavin & $0.022 \mathrm{~h}$ & $0.028 \mathrm{~g}$ & $0.034 \mathrm{f}$ & $0.057 \mathrm{~b}$ & $0.041 \mathrm{de}$ \\
\hline pyhtosterol & $0.003 d$ & $0.006 \mathrm{c}$ & $0.009 a$ & $0.01 \mathrm{a}$ & $0.008 a b$ \\
\hline tannins & $0.02 \mathrm{a}$ & $0.021 \mathrm{c}$ & $0.021 \mathrm{c}$ & $0.023 b$ & $0.021 \mathrm{c}$ \\
\hline coumarins & $0.21 \mathrm{~d}$ & $0.03 a$ & $0.036 \mathrm{c}$ & $0.042 b$ & $0.037 \mathrm{c}$ \\
\hline flavonoids & $0.008 \mathrm{a}$ & $0.007 \mathrm{~b}$ & $0.008 \mathrm{a}$ & $0.009 \mathrm{a}$ & $0.007 \mathrm{~b}$ \\
\hline alkaloids & $0.003 \mathrm{a}$ & $0.003 \mathrm{a}$ & $0.002 \mathrm{~b}$ & $0.002 \mathrm{~b}$ & $0.002 b$ \\
\hline phenols & $0.001 \mathrm{c}$ & $0.001 \mathrm{c}$ & $0.002 \mathrm{~b}$ & $0.003 a$ & $0.002 b$ \\
\hline saponins & $0.002 \mathrm{~d}$ & $0.003 \mathrm{c}$ & $0.003 \mathrm{c}$ & $0.006 a$ & $0.004 \mathrm{~b}$ \\
\hline pectins & $0.002 \mathrm{a}$ & $0.001 \mathrm{~b}$ & $0.002 \mathrm{a}$ & $0.002 \mathrm{a}$ & $0.001 \mathrm{~b}$ \\
\hline triterpenoids & $0.054 \mathrm{a}$ & $0.0051 \mathrm{c}$ & $0.0052 \mathrm{~b}$ & $0.048 d$ & $0.0051 \mathrm{c}$ \\
\hline
\end{tabular}

Data were statistically analyzed through Analysis of Variance and Duncan's Multiple Range Test using DSAASTAT (Onofri, Italy). Each nutritional element was compared along its own quantities extracted with different concentration of organic solvent. Each horizontal change in alphabet represents significant difference calculated at $p \geq 0.05$ Table 2: Nutritional contents $(\mathrm{g} / \mathrm{Kg})$ of barley seeds after application of different sub-fractions extracted from chloroform fraction. 
Citation: Yousaf A, Ashraf Y, Yasin NA, Ibrahim A, Ahmad A, et al. (2016) Analysis of Microbial Biochemical Inducting Nutritional Contents in Barley. J Microb Biochem Technol 8: 395-403. doi: 10.4172/1948-5948.1000315
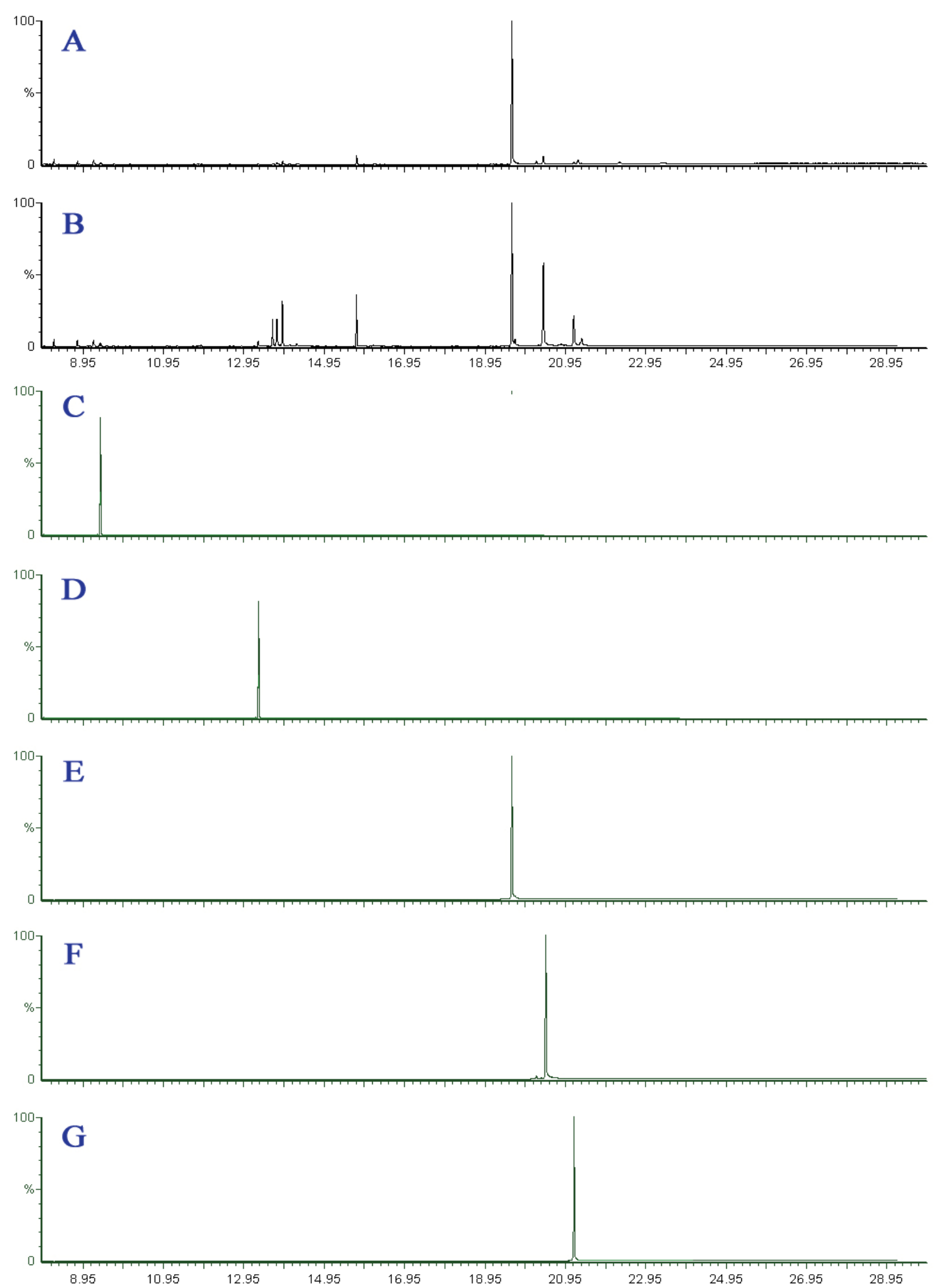

Figure 1: GCMS profiles of two most active chloroform fractions, i.e., $80 \%$ (A) and $100 \%$ (B). Standard run of active compounds were performed providing identical GCMS conditions on same GCMS apparatus. Mevalonic Acid (C), Quinolinic Acid (D), Pyridoxic Acid (E), p-Aminobenzoate (F) and a-Oxobutanoic acid (G). X-axis has been marked with time elapsed for chemical elution and Y-axis represents the height of peaks dependent upon chemical quantities. 
Citation: Yousaf A, Ashraf Y, Yasin NA, Ibrahim A, Ahmad A, et al. (2016) Analysis of Microbial Biochemical Inducting Nutritional Contents in Barley. J Microb Biochem Technol 8: 395-403. doi: 10.4172/1948-5948.1000315

\begin{tabular}{|c|c|c|c|c|c|c|}
\hline & Quinolinic acid & Pyridoxic acid & p-aminobenzoate & $\alpha$-oxobutanoic acid & Mevalonic Acid & Control \\
\hline ascorbic acid & $0.048 \mathrm{~b}$ & $0.012 \mathrm{~d}$ & $0.077 \mathrm{a}$ & $0.051 \mathrm{~b}$ & $0.027 \mathrm{c}$ & $0.028 \mathrm{c}$ \\
\hline niacin & $0.052 \mathrm{~b}$ & $0.043 \mathrm{bc}$ & $0.067 \mathrm{a}$ & $0.037 \mathrm{c}$ & $0.042 \mathrm{bc}$ & $0.023 d$ \\
\hline panthothenic acid & $0.034 \mathrm{a}$ & $0.029 a b$ & $0.034 \mathrm{a}$ & $0.018 c$ & $0.012 \mathrm{~d}$ & $0.017 \mathrm{c}$ \\
\hline pyridoxine & $0.039 \mathrm{~b}$ & $0.019 \mathrm{~cd}$ & $0.065 \mathrm{a}$ & $0.025 \mathrm{c}$ & $0.015 \mathrm{~d}$ & $0.019 \mathrm{~cd}$ \\
\hline thiamine & $0.015 \mathrm{bc}$ & $0.018 \mathrm{~b}$ & $0.028 a$ & $0.009 \mathrm{~cd}$ & $0.015 \mathrm{bc}$ & $0.007 \mathrm{~d}$ \\
\hline folic acid & $0.018 b$ & $0.009 \mathrm{c}$ & $0.035 \mathrm{a}$ & $0.018 b$ & $0.010 \mathrm{c}$ & $0.005 \mathrm{~cd}$ \\
\hline riboflavin & $0.057 \mathrm{a}$ & $0.038 \mathrm{~b}$ & $0.058 a$ & $0.025 \mathrm{c}$ & $0.026 \mathrm{c}$ & $0.021 \mathrm{~cd}$ \\
\hline phytosterol & $0.012 \mathrm{~cd}$ & $0.016 \mathrm{bc}$ & $0.018 b$ & $0.014 \mathrm{c}$ & $0.024 \mathrm{a}$ & $0.016 \mathrm{bc}$ \\
\hline tannins & $0.021 \mathrm{~b}$ & $0.019 \mathrm{c}$ & $0.033 a$ & $0.017 d$ & $0.036 \mathrm{bc}$ & $0.018 \mathrm{~cd}$ \\
\hline coumarins & $0.042 \mathrm{a}$ & $0.039 \mathrm{~b}$ & $0.026 \mathrm{~d}$ & $0.033 \mathrm{c}$ & $0.034 \mathrm{bc}$ & $0.024 \mathrm{e}$ \\
\hline flavonoids & $0.021 \mathrm{~d}$ & $0.033 \mathrm{c}$ & $0.041 \mathrm{a}$ & $0.036 \mathrm{~b}$ & $0.018 \mathrm{e}$ & $0.012 \mathrm{e}$ \\
\hline alkaloids & $0.016 \mathrm{bc}$ & $0.014 \mathrm{c}$ & 0.027 a & $0.018 b$ & $0.017 \mathrm{~b}$ & $0.009 \mathrm{~d}$ \\
\hline phenols & $0.023 a$ & $0.012 \mathrm{~d}$ & $0.017 b$ & $0.009 \mathrm{e}$ & $0.022 \mathrm{a}$ & $0.014 \mathrm{c}$ \\
\hline saponins & $0.009 \mathrm{c}$ & $0.018 b$ & $0.029 a$ & $0.008 \mathrm{c}$ & $0.017 b$ & $0.006 \mathrm{~d}$ \\
\hline pectins & $0.039 a$ & $0.021 \mathrm{c}$ & $0.033 b$ & $0.019 \mathrm{~d}$ & $0.020 \mathrm{c}$ & $0.012 \mathrm{e}$ \\
\hline triterpenoids & $0.053 a$ & $0.027 \mathrm{c}$ & $0.037 \mathrm{bc}$ & $0.039 \mathrm{~b}$ & $0.016 \mathrm{~d}$ & $0.017 \mathrm{~d}$ \\
\hline
\end{tabular}

Data were statistically analyzed through Analysis of Variance and Duncan's Multiple Range Test using DSAASTAT (Onofri, Italy). Each nutritional element was compared along its own quantities extracted after treating plants with different bioactive compounds. Each horizontal change in alphabet represents significant difference calculated at $p \geq 0.05$

Table 3: Quantification of nutritional contents $(\mathrm{g} / \mathrm{Kg})$ of barley seeds harvested from bioactive compounds treated plants.

\begin{tabular}{|c|l|c|c|}
\hline Serial No. & Chemical & Retention Time (m) & Total Peak Area (E) \\
\hline 1 & thymol & 8.03 & 4.7 \\
\hline 2 & eugenol & 8.88 & 4.9 \\
\hline 3 & globulol & 9.17 & 5.1 \\
\hline 4 & mevalonic Acid & 9.29 & 4.3 \\
\hline 5 & quinolinic Acid & 12.33 & 4.7 \\
\hline 6 & E-8-octadecacen-1-ol acetate (ODCA) & 5.6 \\
\hline 7 & pentadenoic Acid & 13.68 & 5.8 \\
\hline 8 & phthalic Ester (Methyl Octyl Ester) & 13.85 & 5.9 \\
\hline 9 & octadecatrienoic Acid (ODTA) & 14.08 & 6.1 \\
\hline 10 & pyridoxic acid & 15.75 & 7.4 \\
\hline 11 & p-aminobenzoate & 19.67 & 6.5 \\
\hline 12 & a-oxobutanoic acid & 20.41 & 5.3 \\
\hline 13 & glycine & 21.18 & 4.2 \\
\hline
\end{tabular}

MZmine (Pluskal, Okinawa, Japan) was used to analyze chromatograms and mass spectra. Properties of each compound were recorded and mentioned against it. Retention time is the time taken by chemicals for their individual elution, and has been calculated in minutes (min); whereas, total peak area has been calculated through MZmine unit of relativity (E)

Table 4: Chemical compounds in chloroform fractions ( $80 \%$ and $100 \%)$ identified through GCMS analysis.

activity of Quinolinic acid and $p$-Aminobenzoate. Quinolinic acid was the second best inducer of vitamins in barley seeds, However, Pyridoxic acid and $\alpha$-Oxobutanoic acid had a variable behavior with respect to each individual vitamin. They could not induce significant quantities of most of the vitamins and other tested nutritional contents, however

\section{Discussion}

Current investigation describes an array of microbial metabolites which affect plant biochemicals. These metabolites more specifically enhance the quantities of those biochemicals which are relevant to nutritional quality of barley produce. Moreover, it also covers the area of plant metabolic processes where the microbial metabolites may take part to influence plant biochemicals. It highlights the exact points in plant metabolic pathways where microbial metabolites may play their role to modulate plant biochemical contents. Therefore, current study broadens the frontiers in plant microbe interaction as well in plant biology.
Solubility of different compounds is extremely variable in a single organic solvent [10]. Smilarly, different organic solvents dissolve highly variable amounts of a single compound [11]. Thus, the optimization of solvent is necessarily required to isolate a desired compound with minimum number of contaminants; and this optimization is imperative when active compound is needed to be isolated from a mixture containing thousands of bacterial metabolites [12]. This study suggests a solvent system of $80 \%$ chloroform and $20 \%$ ethanol to isolate active compounds with minimum number of impurities from bacterial metabolites.

Plants respond to external applications of chemicals, and unprocessed metabolites of $A$. aceti contain a number of compounds with unstudied effects on plants. Hence, the purification of active compounds becomes very important in order to reduce the number of undesired compounds. Furthermore, the composition solvent system affects the quantity and quality of compounds being extracted $[13,14]$. The current study approves the two step extraction of A. aceti 


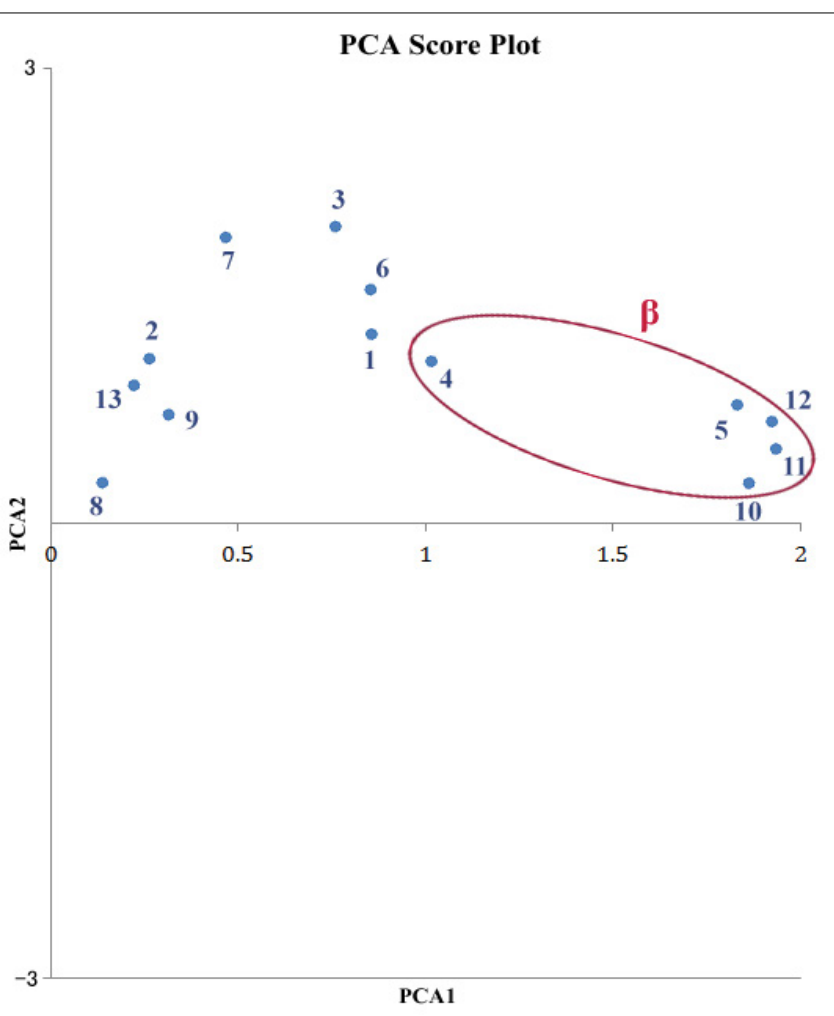

Figure 2: Principle component analysis (PCA) of all detected compounds through GCMS analysis of chloroform subfractions of $A$. aceti extracts. Thirteen chemical componds detected in GCMS analysis were 1: Thymol; 2: Eugenol; 3: Globulol; 4: Mevalonic Acid; 5: Quinolinic Acid; 6: E-8-Octadecacen-1-ol Acetate (ODCA); 7: Pentadenoic Acid; 8: Phthalic Ester; 9: Octadecatrienoic Acid (ODTA); 10: Pyridoxic Acid; 11: p-Aminobenzoate; 12: $\alpha$-Oxobutanoic acid; and 13: Glycine. Association of all compounds was calculated with concentration of chloroform in extraction solvent and induction of vitamins. Statistical package DSAASTAT (Onofri, Italy) was used to perform PCA analysis.

metabolites; first extraction with $100 \%$ chloroform and subsequent extraction with chloroform:ethanol (4:1) solvent system. Thus, resulting extracts have more amounts of active compounds and are more specific in action. Moreover, all the extracted impurities are also chemicals in nature; therefore cannot be multiplied spontaneously unlike the living microbes. Hence, these extracts have no potential pathogenicity toward agricultural crops.

GCMS analysis of biologically active fractions identified the compounds present in them; however, PCA analysis determined the activity of each identified biochemical and arranged them in a hierarchal manner on the basis of their ability to induce vitamins. Four compounds were found to be strongly associated with induction of vitamins; however the interactions among these chemicals have not been studied. It might be possible that they have a cumulative effect on vitamin biosynthesis, or they have synergistic effects on vitamin metabolism [15-17].

All the biochemicals identified in this research are either precursors of vitamin biosynthesis pathways (e.g. Quinolinic Acid and Pyridoxic Acid) or they are facilitators of these pathways (e.g. p-Aminobenzoate and $\alpha$-Oxobutanoic). Thus, the increased production of vitamins in barley can be supported with the logic that 'providing precursors of vitamins would facilitate plants in their production process' $[18,19]$. Moreover, these precursors were in transition state with quantities of vitamins, which means that their higher amounts are more likely shift the reaction balance towards increased quantities of vitamins [20]. PCA analysis categorized the compounds on the basis of their affinity with induced vitamin quantities, and concentration of chloroform in extraction solvent. This revealed the Mevalonic acid as the most active compound among group $\beta$ of identified compounds. Mevalonic acid was the compound with the highest concentration in extracted fraction, as compared to the other compounds of group $\beta$. Moreover, this compound plays a key role in isoprenoid pathway for the biosynthesis of squalene; which is responsible for the ultimate production of sterols. Hence, this study intimates the elevated quantities of squalene and phytosterols in treated barley. However, there is a need of more detailed studies to investigate this aspect.

GCMS analysis also revealed the presence of Glycine in tested fractions. Glycine takes part in the process of Folate biosynthesis for the production of Tetrahydrofolyl poly. It is three step former biochemical reaction just equivalent to the production of 5,10-methenyltetrahydrofolate polyglutamate. Logically, glycine would be in positive relation with vitamins induction; however, it was inversely related with vitamin quantities. These findings indicate some complexities in Folate metabolism, which are still needed to be resolved.

Study concludes that two biochemicals i.e. $p$-Aminobezoate and Quinolinic acid can induce vitamin contents in barley seeds, However, $p$-Aminobezoate is recommended as the best vitamin inducer. All these two biochemicals can be isolated from the metabolites of $A$. aceti using organic solvent system (Chloroform:Ethanol::4:1).

Meanwhile, Current investigation focused on induction of water soluble vitamins in crop (barley) using microbial metabolites. This biological method has no involvement of hazardous chemicals, hence safe for environment. This measure is cost-effective and easy to implement in agricultural fields without any hazard of being a pathogen 

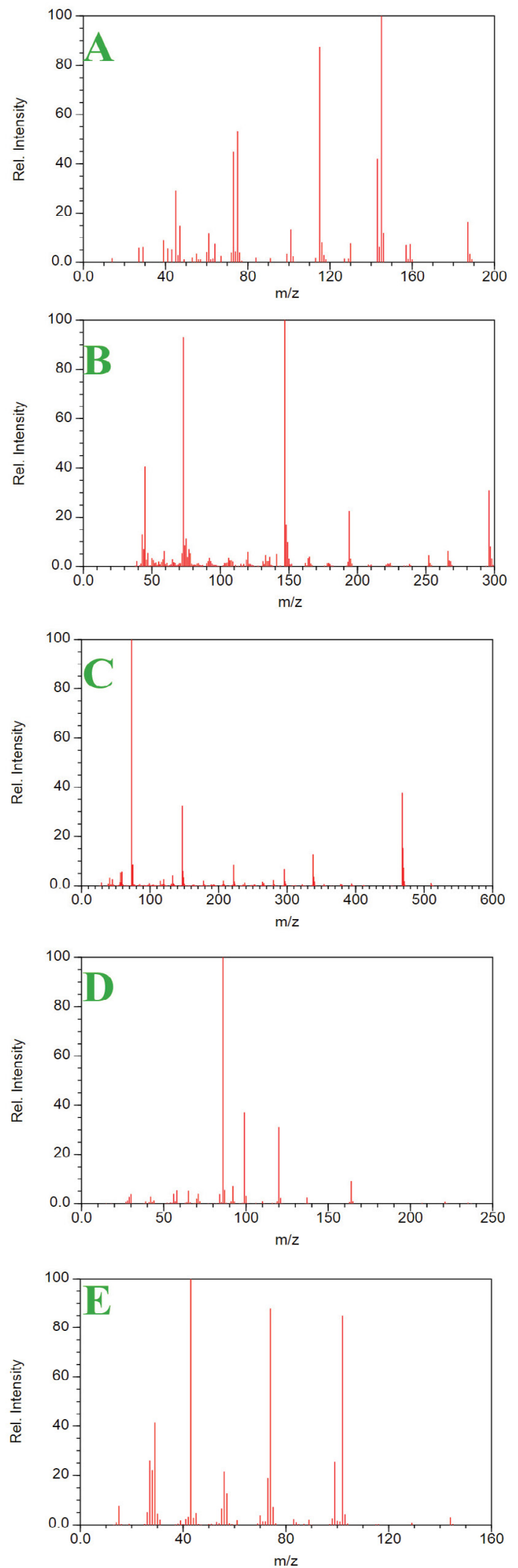
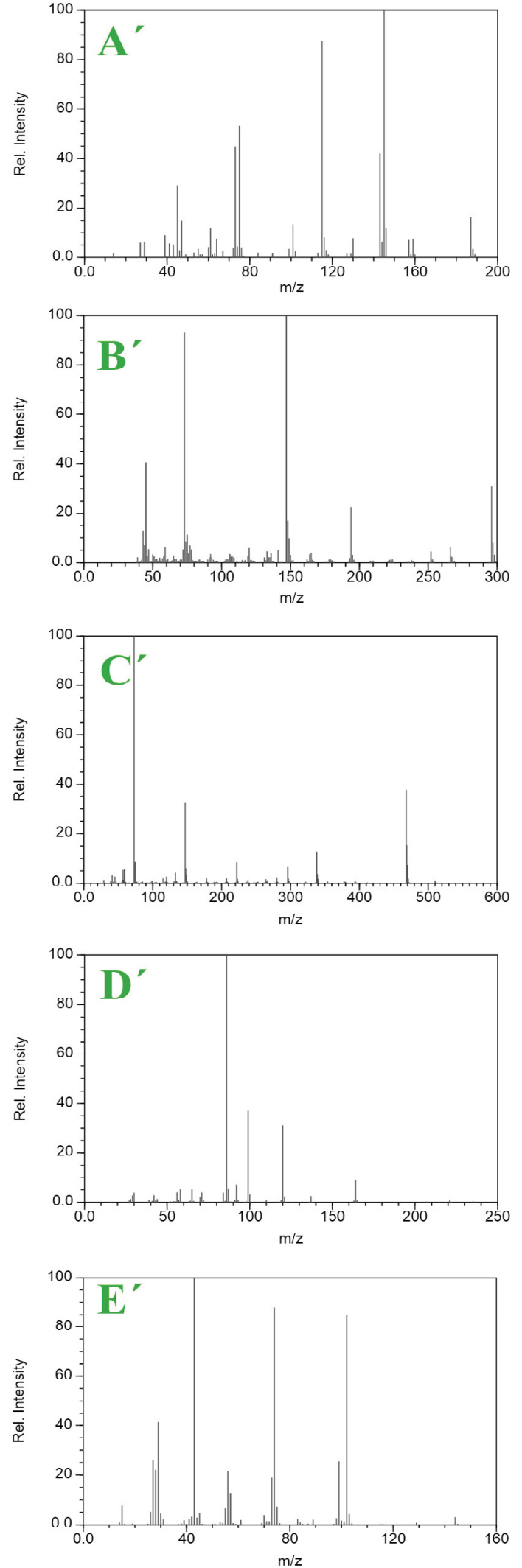

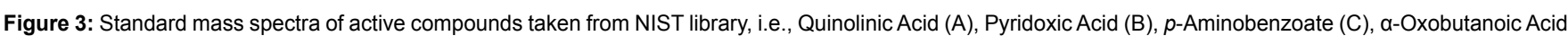
(D) and Mevalonic Acid (E). Mass spectrum recorded during experimental run of $A$. aceti fractions have been tagged with English alphabet followed by symbol -'-. Compounds were identified using TURBOMASS (Perkinelmer, Shelton, USA) equipped with NIST library. 
Citation: Yousaf A, Ashraf Y, Yasin NA, Ibrahim A, Ahmad A, et al. (2016) Analysis of Microbial Biochemical Inducting Nutritional Contents in Barley. J Microb Biochem Technol 8: 395-403. doi: 10.4172/1948-5948.1000315

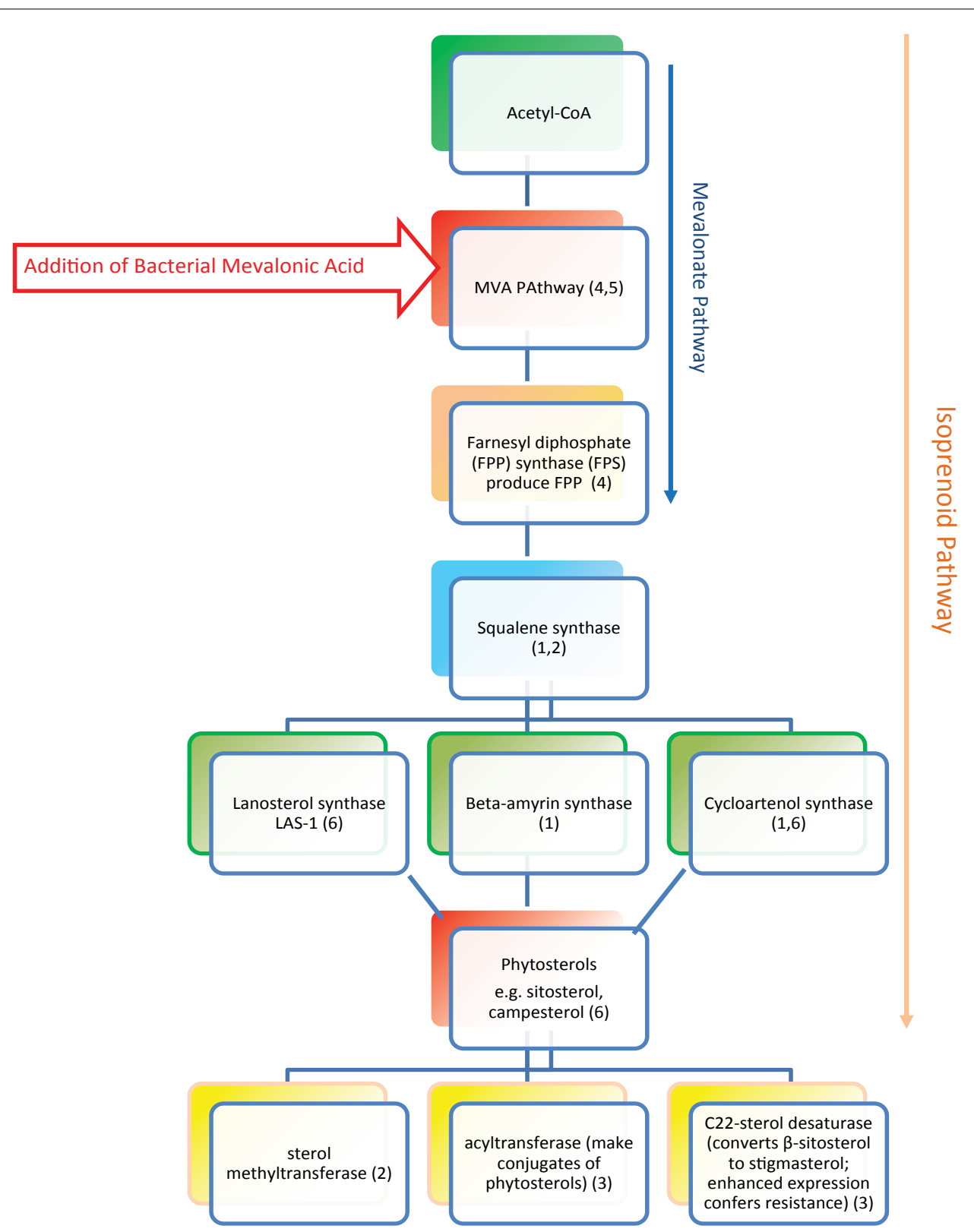

Figure 4: Mechanism of induced quantities of phytosterols and other isoprenoid pathway related compounds.

in future. The technique has a potential to be used for crop and food improvement in Pakistan agricultural system, as well as around the globe.

\section{References}

1. Dong $\mathrm{H}$, Cohen $\mathrm{Y}$ (2001) Extract of killed Penicillium chrysogenum induce resistance against fusarium wilt of melon. Phytoparasitica 29: 421-430.

2. Ragab MMM, Saber MM, El-Morsy SA, Abd El-Aziz ARM (2009) Induction of systemic resistance against root rot of basil using some chemical inducers. Egypt J Phytopathol 37: 59-70.

3. Guijarro B, Melgarejo P, De Cal A (2007) Effect of stabilizers on the shelf-life of Penicillium frequentans conidia and their efficacy as a biological agent against peach brown rot. International Journal of Food Microbiology. 113: 117-124

4. Vazquez G, Melgarejo P De Cal A, Larena I (2013) Persistence, survival, vertical dispersion, and horizontal spread of the biocontrol agent, Penicillium oxalicum strain 212, in different soil types. Applied Soil Ecology 67: 27-36.
5. Ahmad A, Shafique S, Shafique S, Akram W (2014) Penicillium oxalicum directed systemic resistance in tomato against Alternaria alternata. Acta Physiologiae Plantarum 36: 1231-1240

6. Benhamou N, Bélanger RR (1998) Benzothiadiazole-mediated induced resistance to Fusarium oxysporum f. sp. radicis-lycopersici in Tomato. Plant Physiology 118: 1203-1212.

7. Colson-Hanks ES, Deverall BJ (2000) Effect of 2, 6-dichloroisonicotinic acid, its formulation materials and benzothiadiazole on systemic resistance to alternaria leaf spot in cotton. Plant Pathology 49: 171-178.

8. Yousaf A, Qadir A, Anjum T, Ahmad A (2015) Identification of microbia metabolites elevating vitamin contents in barley seeds. Journal of Agricultural and Food Chemistry 63: 7304-7310.

9. Buddenhagen IW, Dull GG (1967) Pink disease of pineapple fruit caused by strains of acetic acid bacteria. Phytopathology 57: 806 .

10. Granberg RA, Rasmuso AC (1999) Solubility of paracetamol in pure solvents. Journal of Chemical \& Engineering Data 44: 1391-1395. 
Citation: Yousaf A, Ashraf Y, Yasin NA, Ibrahim A, Ahmad A, et al. (2016) Analysis of Microbial Biochemical Inducting Nutritional Contents in Barley. J Microb Biochem Technol 8: 395-403. doi: 10.4172/1948-5948.1000315

11. Miller-Chou BA, Koenig JL (2003) A review of polymer dissolution. Progress in Polymer Science 28: 1223-1270.

12. Heryanto R, Hasan M, Abdullah EC, Kumoro AC (2007) Solubility of stearic acid in various organic solventsand its prediction using non-ideal solution models. Science Asia 33: 469-472.

13. Kaufmann B, Christen P (2002) Recent extraction techniques for natural products: microwave-assisted extraction and pressurised solvent extraction. Phytochemical Analysis 13: 105-113.

14. Sultana B, Anwar F, Ashraf M (2009) Effect of extraction solvent/technique on the antioxidant activity of selected medicinal plant extracts. Molecules 14: 2167-2180.

15. Baider A, Cohen Y (2003) Synergistic interaction between BABA and mancozeb in controlling Phytophthora infestans in potato and tomato and Pseudoperonospora cubensis in cucumber. Phytoparasitica 31: 399-409.
16. Edreva A (2004) A novel strategy for plant protection: Induced resistance. Journal of Cell and Molecular Biology 3: 61-69.

17. Brent KJ, Hollomon DW (2007) Fungicide resistance in crop pathogens: How can it be managed? Fungicide Resistance Action Committee, Crop life International, Belgium.

18. Pourcel L, Moulin M, Fitzpatrick TB (2013) Examining strategies to facilitate vitamin $\mathrm{B} 1$ biofortification of plants by genetic engineering. Frontiers in Plant Science 4: 160.

19. Mohammed Y, Lee B, Kang Z, Du G (2014) Development of a two-step cultivation strategy for the production of vitamin B12 by Bacillus megaterium. Microbial Cell Factories 13: 102.

20. Schowen KB, Limbach HH, Denisov GS, Schowen RL (2000) Hydrogen bonds and proton transfer in general-catalytic transition-state stabilization in enzyme catalysis. Biochimica et Biophysica Acta. 1458: 43-62. 